\title{
Analgesia, Anaesthesia and Pregnancy - A Practical Guide - Third Edition
}

\author{
Steve Yentis, Surbhi Malhotra. Cambridge University Press, 2013, 412 pages. \\ ISBN: 978-1-107-60159-8
}

\author{
Branavan Retnasingham, MBBCh • \\ Vit Gunka, MD
}

Received: 31 May 2013/Accepted: 10 June 2013/Published online: 19 June 2013

(C) Canadian Anesthesiologists' Society 2013

Although the insightful cartoons parodying the colourful life of anesthesiologists made famous by Yentis' A View from the Head End are sadly absent, the latest evolution of this established book, edited by two eminent London-based obstetric anesthesiologists, describes several advancements.

In line with previous editions, however, the structure has remained largely unchanged. It still comprises four main sections: 'Preconception and conception', 'Pregnancy', 'Puerperium and after', and 'Organisational aspects'.

The content broadly reflects standard practices in the United Kingdom. Some chapters, such as Prescription and administration of drugs by midwives and Midwifery training, although still applicable in Canada, may be of limited interest to readers in the United States. Most of the book, however, contains core material relevant to any maternity unit.

The layout is clear and the chapter format consistent throughout. It follows an effective formula of including subheadings on Problems/special considerations, Management options, Key points and Further reading. The use of illustrations is economical and reserved primarily for revising neuroanatomy. The illustrations, which are largely schematic, include thoughtful details. The tables contain valuable information but occasionally fail to stand out because of indistinct pale grey formatting.

There has been a subtle reordering of the chapters so they appear in a more logical progression than in the second edition. Examples include epilepsy and convulsions, which now appear as successive chapters, as do rheumatoid arthritis and connective tissue disorders as

B. Retnasingham, MBBCh · V. Gunka, MD ( $₫)$

British Columbia Women's Hospital and Health Centre,

Vancouver, BC, Canada

e-mail: vgunka@cw.bc.ca well as psychiatric disease and substance abuse. It gives an overall impression that more thought has been invested in producing a smoother narrative.

Three new chapters have emerged, entitled Shoulder dystocia, Pneumonia, and Migrants/disadvantaged (featuring women). Also, references at the end of chapters have been updated. Of specific note, the chapter on Physiology of pregnancy now includes a subsection on Haematological changes, an omission in prior editions. The chapter on Maternal mortality has been completely updated to reflect the findings of the latest Centre for Maternal and Child Enquiries report, with a useful summary of obstetric anesthesia-related deaths.

Refreshingly, readers benefit not only from a core of essential knowledge and selected pertinent references but also from highly regarded expert opinion. Moreover, the reader consistently feels as if he or she is having a discussion with a seasoned colleague in the field. This does not detract from its validity but, rather, is a distinctive feature that in many ways contributes to its overall appeal. It is reflective of the aim of providing a hands-on guide.

The theme of practicality continues throughout the book, arising from the need to highlight commonly encountered issues on the maternity unit that have not been inadequately addressed in other available specialist texts whilst simultaneously incorporating topics that focus on patient care and comfort. Dedicated chapters concerning Gastric function and feeding in labour, Bloody tap, Backache, Opioid-induced pruritus, Shivering, and Maternal satisfaction are noteworthy examples.

The authors reflect on the most recent advances in obstetric anesthesia during discussions that focus on topics such as programmed intermittent epidural analgesia or phenylephrine infusion for Cesarean delivery under spinal anesthesia. 
A section on Organisational aspects, which comprises such topics as Antenatal education, Audit, Labour ward organization, Consent, Medicolegal aspects, Record keeping, Minimum standards, Risk management, Postcrisis management and Research on labour ward, ensures that the book avoids being one-dimensional. It also addresses the holistic education of trainees, reflecting the importance of embedding professionalism and governance in training.

The editors argue that not including supporting references for all points made would detract from the readability and usability of the book. However, more of these facts and figures could be specifically referenced without unduly altering the sharp focus of the text by, for example, employing Vancouver-style referencing.

With its design of providing a 'practical' guide aimed at anesthesia trainees, it meets its mandate with elegant efficiency. Its clarity and accessibility makes it of value to a range of allied health professionals. It is not intended to be an alternative to established 'heavyweight' tomes aimed at deep exploration for academic interests and so should not be purchased for this purpose.

Whether acquired as a printed volume or as an eBook, this updated, refreshed, and focused text is a valuable resource. It ensures that both trainees and senior colleagues who are 'occasional' obstetric anesthesiologists are appropriately equipped to negotiate the seemingly unfamiliar and increasingly complex world of obstetric anesthesia.

In the pursuit of quality research one can conduct a prospective, randomized, blinded study. There is no such standardized, approved means to evaluate a book scientifically and compare it to similar texts in the subspecialty. Instead, we have relied on our own innate senses and thus end this critique with our personal opinion: We liked the book!

Competing interests None declared. 